ITO LA; GAION LA; GALATTI FS; BRAZ LT; SANTOS JM. 2014. Resistência de porta-enxertos de cucurbitáceas a nematóides e compatibilidade da enxertia em melão. Horticultura Brasileira 32: 297-302. DOI - http://dx.doi.org/10.1590/S0102-05362014000300010

\title{
Resistência de porta-enxertos de cucurbitáceas a nematóides e compatibilidade da enxertia em melão
}

\section{Letícia A Ito; Lucas A Gaion; Francine S Galatti; Leila T Braz; Jaime M Santos}

${ }^{1}$ UNESP-FCAV, Rod. Prof. Paulo Donato Castellane s/n, 14884-900 Jaboticabal-SP; leleakemi@yahoo.com.br; lucas.gaion@yahoo.com. br; francinegalatti@hotmail.com; leilatb@fcav.unesp.br; jmsantos@fcav.unesp.br

\section{RESUMO}

Devido ao severo ataque de nematoides na cultura do meloeiro rendilhado sob ambiente protegido, este trabalho teve por objetivo selecionar porta-enxertos resistentes a Meloidogyne incognita e $M$. javanica. O experimento foi conduzido em casa de vegetação, de outubro de 2010 a abril de 2011, em Jaboticabal-SP. Foram avaliados 33 porta-enxertos; melões: CNPH 01-930 (Cucumis melo var. flexuosus), CNPH 01-962, CNPH 01-963 (Cucumis melo var. conomon), cvs. Gaúcho Redondo, Gaúcho Comprido, Redondo Amarelo, Gulfcoast, Chilton, Bônus $n^{\circ}$ 2, Fantasy; melancias: cv. Charleston Gray, Progênie da Coréia (Citrullus lanatus); abóboras: cvs. Mra. Ma, Ornamental, Howden, Mammoth, Kururu, Goianinha (Cucurbita moschata); cabaça: Abóbora de Porco, cvs. Maranhão, Brasileirinha (Lagenaria siceraria); moranga: cv. Pataca Gigante (Cucurbita maxima); pepinos: cvs. Caipira, Branco Meio-Comprido, Curumim (Cucumis sativus); buchas: Metro, Semente Branca, Semente Preta (Luffa cylindrica); abóbora d'água (Benincasa hispida); abóbora porta-enxerto: Híbrido cv. Keij; quiabo de Metro (Trichosanthes cucumerins) e Cruá (Sicana odorifera). Para avaliar a resistência de plantas, as mudas foram transplantadas para vasos e inoculados com 3.000 ovos e juvenis de M. incognita via sistema radicular. Aos 50 dias após a inoculação, foi realizada avaliação da resistência das plantas, com base no fator de reprodução do nematoide. Foram realizados os mesmos procedimentos para as duas espécies de nematoides. Nos porta-enxertos resistentes aos nematoides foram realizadas enxertias, com os melões rendilhados 'Bônus n 2' e 'Fantasy'. CNPH 01-962, CNPH 01-963 e melão 'Gaúcho Redondo' foram resistentes a $M$. incognita. Melão 'Redondo Amarelo', melancia 'Charleston Gray', Progênie da Coréia, e Trichosanthes cucumerins, foram resistentes a M. javanica. Benincasa hispida foi resistente a ambas as espécies. As compatibilidades de enxertia entre os porta-enxertos resistentes e os melões rendilhados 'Bônus n 2' e 'Fantasy' foram superiores a 98\%.

Palavras-chave: Cucumis melo var. reticulatus, nematoides, 'Bônus no 2', 'Fantasy'.

\begin{abstract}
Resistance of cucurbita rootstocks to nematodes and compatibility of grafting with net melon
\end{abstract}

Nematodes severely attack net melon plants under protected cultivation conditions. The objective of this research was to select rootstocks with resistance to Meloidogyne incognita and M. javanica. The experiment was carried out under greenhouse conditions from October 2010 to April 2011 in Jaboticabal, São Paulo state, Brazil. Thirty-three cucurbitaceous genotypes were investigated as rootstocks; melons: CNPH 01-930 (Cucumis melo var. flexuosus), CNPH 01-962, 01-963 CNPH (Cucumis melo var. conomon), cvs. Gaucho Redondo, Gaucho Comprido, Redondo Amarelo, Gulfcoast, Chilton, Bônus no. 2, Fantasy; watermelons: cv. Charleston Gray, Progênie da Coréia (Citrullus lanatus); pumpkins: cvs. Mra. Ma, Ornamental, Howden, Mammoth, Kururu, Goianinha (Cucurbita moschata); gourd: Abóbora de Porco, cvs. Maranhão, Brasileirinha (Lagenaria siceraria); squash: cv. Pataca Gigante (Cucurbita maxima); cucumber: cvs. Caipira, Branco Meio Comprido, Curumim (Cucumis sativus); loofah: Metro, Semente Branca, Semente Preta (Luffa cylindrica); wax gourd (Benincasa hispida); pumpkin rootstock: Hybrid cv. Keij; snake gourd (Trichosanthes cucumerins) and musk cucumber (Sicana odorifera). To evaluate the resistance, seedlings were transplanted to pots and the root inoculated with 3,000 eggs and second stage juveniles of $M$. incognita and $M$. javanica. Fifty days after the inoculation, the plants were evaluated for nematode resistance by means of the reproduction factor. The grafting compatibility between net melon cvs. Bonus no. 2 and Fantasy and the rootstocks previously characterized as resistant were evaluated by means of 60 graftings. CNPH 01-962, CNPH 01-963 and melon 'Gaucho Redondo', were considered resistant to M. incognita. Melon 'Redondo Amarelo', watermelon 'Charleston Gray', watermelon Progênie da Coréia, Trichosanthes cucumerins were considered resistant to M. javanica. Benincasa hispida was resistant to $M$. javanica and $M$. incognita. The compatibility between net melons and resistant rootstocks was higher than $98 \%$.

Keywords: Cucumis melo var. reticulatus, nematodes, Bônus II, Fantasy.

(Recebido para publicação em 31 de janeiro de 2013; aceito em 6 de junho de 2014) (Received on January 31, 2013; accepted on June 6, 2014)

$\mathrm{O}$ cultivo intensivo de cucurbitáceas tem promovido o desenvolvimento de nematoides que promovem prejuízos significativos em cultivos de alta infestação (Pinheiro \& Amaro, 2010). O principal sintoma dos nematoides do grupo Meloidogyne é a presença de galhas no sistema radicular resultando num lento desenvolvimento da planta, pois dificultam a absorção de água e nutrientes, causam declínio na qualidade e até perdas totais da produção (Chandra 
et al., 2010). Na parte aérea das plantas atacadas, normalmente as folhas ficam cloróticas, reduzem de tamanho e quantidade de folhas e pode ocorrer murcha da planta nas horas mais quentes do dia. Em interação com outros patógenos como fungos e bactérias, podem causar podridão radicular e murcha (Pinheiro \& Amaro, 2010).

Siguenza et al. (2005) relataram que Meloidogyne incognita e $M$. javanica são as espécies de nematoides de galhas que predominam na cultura do meloeiro na Califórnia e, mesmo com baixa população, têm ocasionado grandes perdas.

A utilização de plantas resistentes é o método mais econômico no controle dos nematoides de galhas. Porém, no Brasil, todas as cultivares de melão, melancia e abóboras são suscetíveis a Meloidogyne spp. A espécie Cucumis metuliferus é altamente resistente a Meloidogyne hapla, $M$. incognita, $M$. javanica e $M$. arenaria, mas o cruzamento desta com Cucumis spp. visando a obtenção de híbridos resistentes, produtivos e com qualidade, não tem apresentado resultados satisfatórios. (Pinheiro \& Amaro, 2010).

A enxertia de cultivares de melão em porta-enxertos de cucurbitáceas resistentes a nematoides é considerada uma prática alternativa aos nematoides de galhas (Thies et al., 2010). O interesse por esta técnica vem crescendo pelo mundo, pois além das vantagens de resistência e/ou tolerância a altas e baixas temperaturas e nematoides, determinados porta-enxertos também minimizam a absorção de resíduos agroquímicos indesejáveis (Lee et al., 2010).

$\mathrm{Na}$ Itália, a utilização de porta-enxertos híbridos resistentes, representa o principal instrumento de prevenção de doenças na cultura do meloeiro (Martignoni et al., 2011). O número de produtores de mudas enxertadas comerciais tem aumentado significativamente pelo mundo, reflexo do aumento da preferência dos agricultores por este tipo de muda (Lee et al., 2010).

A utilização de porta-enxertos de Lagenaria siceraria e híbridos de abóbora foi descrita como método não químico aceitável no controle de Meloidogyne spp. na cultura do pepino, em casa de vegetação(Ban et al., 2011). Segundo os mesmos autores, a enxertia pode ser o método mais adequado para pequenos produtores que não adotam sistema hidropônico de cultivo, por ser mais oneroso.

Em ambiente protegido, os problemas de contaminação por patógenos de solo estão cada vez mais difíceis de solucionar por métodos tradicionais de controle. A busca por porta-enxertos de cucurbitáceas resistentes é uma alternativa para a diminuição da incidência de nematoides (Pinheiro \& Amaro, 2010).

Porta-enxertos pertencentes a espécies diferentes muitas vezes são preferidos por causa da diversidade genética (Lee et al., 2010). A cucurbitácea $C$. metuliferus pode ser usada como porta-enxerto para melão, com a intenção de prevenir o crescimento da população de M. incognita (Siguenza et al., 2005).

Pesquisas recentes estão cada vez mais voltadas para o desenvolvimento de tecnologias para enxertia em associação com porta-enxertos eficientes no controle de doenças (Lee et al., 2010). Estudos sobre comportamento, compatibilidade, produtividade, resistência e/ou tolerância dos porta-enxertos e enxertos, bem como avaliações de espécies de porta-enxertos sob diferentes condições ambientais são fundamentais para a escolha de porta-enxerto adequado, que combine resistência e produtividade (Goto et al., 2003). Diante do exposto, este trabalho teve por objetivo selecionar porta-enxertos resistentes a $M$. incognita e/ou M. javanica e verificar a compatibilidade da enxertia destes porta-enxertos com meloeiro rendilhado.

\section{MATERIAL E MÉTODOS}

Os experimentos foram conduzidos em casa de vegetação da UNESP-FCA, em Jaboticabal-SP, de outubro de 2010 a abril de 2011.

As temperaturas médias mensais durante os experimentos foram estáveis, sem grandes variações: outubro $23^{\circ} \mathrm{C}$; novembro $24,1^{\circ} \mathrm{C}$; dezembro $25,1^{\circ} \mathrm{C}$ (experimento de 2010); janeiro $24,7^{\circ} \mathrm{C}$; fevereiro $24,6^{\circ} \mathrm{C}$; março $24,6^{\circ} \mathrm{C}$ (experimento de 2011).

$\mathrm{O}$ inóculo inicial foi proveniente de plantas de tomateiros 'Kada' infectados por $M$. incognita e $M$. javanica separa- dos, oriundos do Setor de Olericultura e Plantas Aromático-Medicinais, da UNESP-FCAV, preparado segundo a técnica de Hussey \& Barker (1973), com as modificações feitas por Bonetti \& Ferraz (1981) em que as raízes das plantas de tomateiro infectado com o nematoide são lavadas cuidadosamente para retirar o solo aderido; posteriormente são picadas em pedaços de aproximadamente dois cm de comprimento e triturados com auxílio de um liquidificador contendo solução de hipoclorito de sódio a $0,5 \%$ durante 30 segundos. A suspensão obtida foi passada em peneira de 200 mesh sobre a peneira de 500 mesh, com auxílio de uma piseta contendo água; o excesso de hipoclorito foi removido. A primeira peneira reteve as raízes trituradas, que foram descartadas, e a segunda peneira reteve os ovos e juvenis de segundo estádio. A estimativa da população de ovos e juvenis de segundo estádio na suspensão foi efetuada com auxílio da câmara de contagem de Peters, no microscópio e a concentração da suspensão foi ajustada para 300 ovos e juvenis de segundo estádio $\mathrm{mL}^{-1}$. Ambas as espécies foram identificadas com base nos caracteres morfológicos do padrão perineal, preparado conforme Taylor \& Netscher (1974), conforme citado em Hartman \& Sasser (1985) e na morfologia da região labial dos machos (Eisenback et al., 1981).

Para a multiplicação e manutenção do inóculo inicial dos nematoides, foram utilizadas plantas de berinjela, cujas mudas foram obtidas por meio de semeadura em bandeja de poliestireno expandido, com 128 células, contendo substrato Bioplant ${ }^{\circledR}$ para hortaliças de fruto. Após 30 dias da semeadura, as mudas foram transplantadas para vasos de cerâmica com capacidade de três litros. Os vasos foram preenchidos com mistura de terra, esterco de curral seco e areia, na proporção de $1: 1: 1$, previamente autoclavado $\left(120^{\circ} \mathrm{C}\right.$ a 1 atm por 1 hora). No momento do transplantio foi feita a inoculação com $10 \mathrm{~mL}$ da suspensão contendo 3.000 ovos e juvenis de segundo estádio de Meloidogyne incognita e de M. javanica, sendo cada espécie inoculada em lotes separados de vasos.

O delineamento foi de blocos ao 
acaso com 33 tratamentos (genótipos de cucurbitáceas) e dez repetições, cada repetição composta por um vaso contendo uma planta inoculada para cada uma das espécies de nematoides.

Os genótipos de cucurbitáceas testados foram os melões: CNPH 01-930, CNPH 01-962, CNPH 01963, 'Gaúcho Redondo', 'Gaúcho Comprido', 'Redondo Amarelo', 'Gulfcoast', 'Chilton', 'Bônus n’ 2', 'Fantasy' (dois últimos utilizados como testemunhas não enxertados); as melancias: 'Charleston Gray', Progênie da Coréia (Citrullus lanatus); as abóboras: 'Mra. Ma', 'Ornamental', 'Howden', 'Mammoth', 'Kururu', 'Goianinha'; cabaça: Abóbora de Porco, 'Maranhão', 'Brasileirinha' (Lagenaria siceraria); a moranga: 'Pataca Gigante' (Cucurbita maxima); os pepinos: 'Caipira', 'Branco Meio-Comprido', 'Curumim' (Cucumis sativus); buchas: Metro, Semente Branca, Semente Preta (Luffa cylindrica); abóbora d'água (Benincasa hispida); abóbora portaenxerto híbrido 'Keij' $\mathrm{F}_{1}$; Quiabo de Metro (Trichosanthes cucumerins) e Cruá (Sicana odorifera).

As sementes dos acessos foram semeadas em bandeja de poliestireno expandido, com 128 células, contendo substrato comercial para hortaliças de fruto. Quinze dias após a semeadura, as mudas foram transplantadas para vasos de cerâmica com capacidade de três litros, contendo uma mistura de solo, esterco de curral e areia, na proporção de 1:1:1 previamente autoclavada $\left(120^{\circ} \mathrm{C}\right.$, a 1 atm., por 1 hora).

Após quatro dias do transplantio, foi realizada a inoculação de ovos e juvenis de segundo estádio das duas espécies de nematoides em dez mudas de cada genótipo. Para tal, foi aplicado por meio de pipeta graduada, $10 \mathrm{~mL}$ da suspensão por repetição, ou seja, 300 ovos e juvenis de segundo estádio $\mathrm{mL}^{-1}$. Portanto a população inicial (PI) foi de 3000 ovos e juvenis de segundo estádio por vaso. Para o preparo da suspensão de ovos e juvenis de segundo estádio, foi utilizada a técnica de Hussey \& Barker (1973) modificada por Bonetti \& Ferraz (1981), utilizando-se das raízes de berinjela previamente infectadas.

Aos 50 dias após a inoculação, a par- te aérea do porta-enxerto foi descartada, e as raízes lavadas. As raízes foram individualmente trituradas em água com hipoclorito de sódio a $0,5 \%$, na proporção $3: 1$, com auxílio de um liquidificador por 30 segundos. Logo após, o conteúdo foi despejado sobre peneiras de 200 e 500 mesh sobrepostas. O conteúdo da peneira de 200 mesh foi descartado e o conteúdo da peneira de 500 mesh foi transferido para um frasco e mantido em geladeira para posterior contagem da população final (PF), com auxílio da câmara de contagem de Peters.

A resistência dos genótipos a $M$. incognita foi avaliada por meio da média do Fator de Reprodução (FR) obtido em cada sistema radicular (Oostenbrink, 1966), no qual a população final (PF) de nematoides foi dividida pelo número de ovos e juvenis de segundo estádio inoculados por planta, ou seja, a população inicial (PI), de modo a se obter os valores médios de FR para cada genótipo. Foram considerados resistentes aos nematoides, os genótipos com valores de $\mathrm{FR}<1$ e suscetíveis os que apresentaram valores de $\mathrm{FR} \geq 1$.

A seleção dos porta-enxertos resistentes ocorreu no período de outubro a dezembro de 2010 e foi repetido no período de janeiro a março de 2011 para comprovação da resistência dos genótipos de cucurbitáceas.

A verificação da compatibilidade entre porta-enxerto resistente e enxerto de melão rendilhado ocorreu em abril de 2011. Foi realizada a semeadura dos porta-enxertos e dos enxertos ('Bônus $\mathrm{n}^{\circ} 2$ ' e 'Fantasy') em bandejas de poliestireno expandido com 128 células preenchidas com substrato comercial. A data de semeadura dos porta-enxertos e dos enxertos foi feita de acordo com o período de dias para emergência e desenvolvimento da planta de cada genótipo, de maneira a atingir o ponto ideal de enxertia concomitantemente. $\mathrm{O}$ período para atingir o ponto ideal de enxertia, de cada genótipo foi verificado durante a semeadura e emergência em outros experimentos com as mesmas cucurbitáceas pelos mesmos autores. Desse modo, a semeadura dos genótipos ocorreu em diferentes datas. Em 05 de abril de 2011 foram semeados melão CNPH 01-962, CNPH 01-963 e $B$. hispida; em 08 de abril de 2011, melão 'Gaúcho Redondo', melão 'Redondo Amarelo', melancia 'Charleston Gray', melancia Progênie Coreia, melão 'Gulfcoast' e melão 'Chilton'; em 11 de abril de 2011: melão rendilhado 'Bônus n'2' e 'Fantasy'; em 13 de abril de 2011, melão CNPH 01-930 e L. siceraria; em 15 de abril de 2011, abóbora 'Goianinha', abóbora 'Brasileirinha', abóbora 'Maranhão' e bucha Metro; e em 19 de abril de 2011, T. cucumeirns (quiabo de Metro) e Híbrido 'Keij' $\mathrm{F}_{1}$.

A enxertia ocorreu 23 dias após a primeira data de semeadura. A enxertia foi do tipo fenda-cheia e foram realizadas quando as mudas apresentavam a segunda folha em inicio de crescimento.

As mudas enxertadas foram colocadas em copos plásticos furados com capacidade de $200 \mathrm{~mL}$ preenchidos com substrato comercial Bioplant ${ }^{\circledR}$ e mantidas em câmara úmida (constituída por uma "piscina" de $1 \mathrm{~m}$ de largura, $7 \mathrm{~m}$ de comprimento e $0,8 \mathrm{~m}$ de altura, coberta com polietileno transparente e tela de sombreamento dentro de uma casa de vegetação) até a cicatrização da região enxertada.

A compatibilidade foi obtida pela porcentagem de mudas enxertadas vivas após a cicatrização do local enxertado. $\mathrm{O}$ delineamento experimental das enxertias foi de blocos ao acaso, sendo a parcela constituída por dez plantas enxertadas, com nove tratamentos e seis repetições, totalizando 60 enxertias com o melão rendilhado 'Bônus $n^{\circ} 2$ ' e 60 enxertias com o melão rendilhado 'Fantasy' em cada porta-enxerto considerado resistente às duas ou a uma das espécies de nematoides.

\section{RESULTADOS E DISCUSSÃO}

Com base na classificação proposta por Oostenbrink (1966) em que se utiliza o fator de reprodução para determinar a resistência a nematoides, no experimento de 2010, as cucurbitáceas: CNPH 01-930, CNPH 01-962, CNPH 01-963, melão 'Gaúcho redondo', e Benincasa hispida foram consideradas resistentes a Meloidogyne incognita, já que apresentaram $\mathrm{FR}<1$; os demais materiais foram considerados suscetíveis por apresenta- 
Tabela 1. Médias do Fator de Reprodução (FR) e Classificação (CL) de Meloidogyne incognita e Meloidogyne javanica dos 31 genótipos de cucurbitáceas e dois melões rendilhados em 2010 e em 2011 [average of reproduction factor (FR) and resistance classification (CL) of M. incognita and M. javanica from 31 genotypes of cucurbits and two net melons from 2010 and 2011). Jaboticabal, UNESP, 2011.

\begin{tabular}{|c|c|c|c|c|c|c|c|c|c|}
\hline \multirow{3}{*}{ Tratamentos } & \multirow{3}{*}{ Nome científico } & \multicolumn{4}{|c|}{ M. incognita } & \multicolumn{4}{|c|}{ M. javanica } \\
\hline & & \multicolumn{2}{|c|}{2010} & \multicolumn{2}{|c|}{2011} & \multicolumn{2}{|c|}{2010} & \multicolumn{2}{|c|}{2011} \\
\hline & & FR* & $\mathbf{C L}$ & FR* $^{*}$ & CL & FR* & CL & FR* & $\mathbf{C L}$ \\
\hline CNPH 01-930 & Cucumis melo var. flexuosus & 0 & $\mathrm{R}$ & $1,0 \mathrm{i}$ & $\mathrm{S}$ & $1,4 \mathrm{o}$ & $\mathrm{S}$ & $16,7 \mathrm{c}$ & $\mathrm{S}$ \\
\hline CNPH 01-962 & Cucumis melo var. conomon & 0 & $\mathrm{R}$ & $0,3 \mathrm{j}$ & $\mathrm{R}$ & $1,1 \mathrm{p}$ & $\mathrm{S}$ & $17,3 \mathrm{~b}$ & $\mathrm{~S}$ \\
\hline CNPH 01-963 & Cucumis melo var. conomon & 0 & $\mathrm{R}$ & $0,2 \mathrm{j}$ & $\mathrm{R}$ & $1,1 \mathrm{p}$ & S & $15,4 \mathrm{~d}$ & $\mathrm{~S}$ \\
\hline melão 'Gaúcho Redondo’ & Cucumis melo & 0 & $\mathrm{R}$ & $0,7 \mathrm{j}$ & $\mathrm{R}$ & $2,5 \mathrm{~g}$ & $\mathrm{~S}$ & 19,9 a & $\mathrm{S}$ \\
\hline melão ‘Gaúcho Caipira’' & Cucumis melo & $17,4 \mathrm{c}$ & S & $2,6 \mathrm{~g}$ & S & 1,81 & S & $6,5 \mathrm{f}$ & S \\
\hline melão ‘Redondo Amarelo’ & Cucumis melo & $18,1 \mathrm{~b}$ & $\mathrm{~S}$ & $3,6 \mathrm{f}$ & $\mathrm{S}$ & $0,6 \mathrm{~s}$ & $\mathrm{R}$ & $0,5 \mathrm{w}$ & $\mathrm{R}$ \\
\hline melão 'Gulfcoast' & Cucumis melo var. reticulatus & $16,7 \mathrm{c}$ & S & $4,7 \mathrm{c}$ & S & $0,3 \mathrm{u}$ & $\mathrm{R}$ & $1,0 \mathrm{u}$ & S \\
\hline melão 'Chilton' & Cucumis melo var. reticulatus & $25,1 \mathrm{a}$ & $\mathrm{S}$ & $3,4 \mathrm{e}$ & $\mathrm{S}$ & $0,5 \mathrm{t}$ & $\mathrm{R}$ & $1,0 \mathrm{u}$ & $\mathrm{S}$ \\
\hline melancia 'Charleston Gray' & Citrullus lanatus & 1,01 & $\mathrm{~S}$ & $4,4 \mathrm{~d}$ & S & $0,1 \mathrm{v}$ & $\mathrm{R}$ & $0,9 \mathrm{u}$ & $\mathrm{R}$ \\
\hline melancia Progênie Coréia & Citrullus lanatus & $2,6 \mathrm{j}$ & $\mathrm{S}$ & $2,3 \mathrm{~g}$ & $\mathrm{~S}$ & $0,9 \mathrm{q}$ & $\mathrm{R}$ & $0,4 \mathrm{w}$ & $\mathrm{R}$ \\
\hline abóbora 'Mra. Ma' & Cucurbita moschata & $7,1 \mathrm{~d}$ & S & $2,0 \mathrm{~h}$ & S & $1,6 \mathrm{~m}$ & S & $2,1 \mathrm{p}$ & S \\
\hline abóbora 'Ornamental' & Cucurbita moschata & $2,2 \mathrm{j}$ & $\mathrm{S}$ & $2,0 \mathrm{~h}$ & $\mathrm{~S}$ & $2,5 \mathrm{~g}$ & $\mathrm{~S}$ & $1,5 \mathrm{r}$ & $\mathrm{S}$ \\
\hline abóbora 'Howden’ & Cucurbita moschata & $2,7 \mathrm{j}$ & $\mathrm{S}$ & $1,2 \mathrm{i}$ & S & 1,3 o & $\mathrm{S}$ & $2,6 \mathrm{n}$ & $\mathrm{S}$ \\
\hline abóbora 'Mammoth' & Cucurbita moschata & $2,4 j$ & $\mathrm{~S}$ & $1,2 \mathrm{i}$ & $\mathrm{S}$ & $1,6 \mathrm{~m}$ & $\mathrm{~S}$ & $1,1 \mathrm{t}$ & $\mathrm{S}$ \\
\hline abóbora 'Kururu' & Cucurbita moschata & $3,2 \mathrm{i}$ & S & $1,4 \mathrm{i}$ & S & $4,5 \mathrm{c}$ & S & $5,0 \mathrm{i}$ & S \\
\hline abóbora 'Goianinha' & Cucurbita moschata & $4,8 \mathrm{~h}$ & $\mathrm{~S}$ & $2,2 \mathrm{~h}$ & $\mathrm{~S}$ & $1,4 \mathrm{o}$ & $\mathrm{S}$ & $2,1 \mathrm{p}$ & $\mathrm{S}$ \\
\hline cabaça & Lagenaria siceraria & $5,2 \mathrm{~g}$ & $\mathrm{~S}$ & $1,1 \mathrm{i}$ & $\mathrm{S}$ & $0,8 \mathrm{r}$ & $\mathrm{R}$ & $1,0 \mathrm{u}$ & $\mathrm{S}$ \\
\hline abóbora de porco & Cucurbita moschata & $3,5 \mathrm{i}$ & S & $1,3 \mathrm{i}$ & S & $2,1 \mathrm{j}$ & S & $1,2 \mathrm{t}$ & S \\
\hline abóbora 'Brasileirinha' & Cucurbita moschata & $7,6 \mathrm{~d}$ & S & $1,2 \mathrm{i}$ & S & $2,8 \mathrm{f}$ & S & $2,4 \mathrm{o}$ & S \\
\hline abóbora 'Maranhão' & Cucurbita moschata & $5,3 \mathrm{~g}$ & $\mathrm{~S}$ & $1,8 \mathrm{~h}$ & $\mathrm{~S}$ & $2,4 \mathrm{~h}$ & $\mathrm{~S}$ & $2,9 \mathrm{~m}$ & $\mathrm{~S}$ \\
\hline moranga 'Pataca Gigante' & Cucurbita maxima & $2,8 \mathrm{j}$ & $\mathrm{S}$ & $2,0 \mathrm{~h}$ & $\mathrm{~S}$ & $1,9 \mathrm{k}$ & $\mathrm{S}$ & $1,1 \mathrm{t}$ & $\mathrm{S}$ \\
\hline pepino 'Caipira' & Cucumis sativus & $6,7 \mathrm{e}$ & S & $2,8 \mathrm{~g}$ & S & $3,5 \mathrm{~d}$ & S & $1,6 \mathrm{q}$ & S \\
\hline pepino 'B. Meio-Comprido' & ' Cucumis sativus & $5,9 \mathrm{f}$ & S & $7,5 \mathrm{a}$ & S & $3,3 \mathrm{e}$ & S & $5,3 \mathrm{~h}$ & S \\
\hline pepino híbrido 'Curumin' & Cucumis sativus & $1,4 \mathrm{k}$ & $\mathrm{S}$ & $4,2 \mathrm{~d}$ & $\mathrm{~S}$ & $5,3 \mathrm{a}$ & $\mathrm{S}$ & $5,9 \mathrm{~g}$ & $\mathrm{~S}$ \\
\hline bucha Metro & Luffa cylindrica & $1,9 \mathrm{k}$ & $\mathrm{S}$ & $1,4 \mathrm{i}$ & $\mathrm{S}$ & $0,7 \mathrm{~s}$ & $\mathrm{R}$ & $1,0 \mathrm{u}$ & $\mathrm{S}$ \\
\hline bucha Semente Branca & Luffa cylindrica & $3,2 \mathrm{i}$ & $\mathrm{S}$ & $6,1 \mathrm{~b}$ & $\mathrm{~S}$ & $1,6 \mathrm{~m}$ & $\mathrm{~S}$ & $1,3 \mathrm{~s}$ & $\mathrm{~S}$ \\
\hline bucha Semente Preta & Luffa cylindrica & $3,4 \mathrm{i}$ & $\mathrm{S}$ & $1,1 \mathrm{i}$ & $\mathrm{S}$ & $1,5 \mathrm{n}$ & $\mathrm{S}$ & 3,21 & $\mathrm{~S}$ \\
\hline abóbora d'água & Benincasa hispida & 0,41 & $\mathrm{R}$ & $0,1 \mathrm{j}$ & $\mathrm{R}$ & $0,8 \mathrm{r}$ & $\mathrm{R}$ & $0,3 \mathrm{w}$ & $\mathrm{R}$ \\
\hline quiabo de Metro & Trichosanthes cucumerins & $1,2 \mathrm{k}$ & $\mathrm{S}$ & $1,9 \mathrm{~h}$ & $\mathrm{~S}$ & $0,1 \mathrm{v}$ & $\mathrm{R}$ & $0,6 \mathrm{v}$ & $\mathrm{R}$ \\
\hline cruá & Sicana odorifera & $1,5 \mathrm{k}$ & $\mathrm{S}$ & $3,3 \mathrm{f}$ & $\mathrm{S}$ & $2,2 \mathrm{i}$ & $\mathrm{S}$ & $10,1 \mathrm{e}$ & $\mathrm{S}$ \\
\hline porta-enxerto 'Keij' $F_{1}$ & Cucurbita moschata & $4,2 \mathrm{~h}$ & $\mathrm{~S}$ & $5,0 \mathrm{c}$ & $\mathrm{S}$ & $4,8 \mathrm{~b}$ & $\mathrm{~S}$ & $4,7 \mathrm{j}$ & $\mathrm{S}$ \\
\hline melão rendilhado 'Bônus n ${ }^{\circ}$ '' & ' Cucumis melo var. reticulatus & $4,6 \mathrm{~h}$ & $\mathrm{~S}$ & $2,0 \mathrm{~h}$ & $\mathrm{~S}$ & $5,3 \mathrm{a}$ & $\mathrm{S}$ & $3,7 \mathrm{k}$ & $\mathrm{S}$ \\
\hline melão rendilhado 'Fantasy' & Cucumis melo var. reticulatus & $6,6 \mathrm{e}$ & $\mathrm{S}$ & $2,4 \mathrm{~g}$ & $\mathrm{~S}$ & $2,8 \mathrm{f}$ & $\mathrm{S}$ & $5,2 \mathrm{~h}$ & $\mathrm{~S}$ \\
\hline CV (\%) & & 12,5 & & 22,0 & & 3,9 & & 3,4 & \\
\hline
\end{tabular}

Médias seguidas da mesma letra na coluna não diferem entre si pelo teste de Scott \& Knott, $(\mathrm{p}<0,05)$ [means followed by the same letter at column did not differ by Scott \& Knott test, $\mathrm{p}<0,05)]$. ${ }^{*} \mathrm{FR}<1,0=$ resistente (R); FR $\geq 1,0=$ suscetível (S) (Oostenbrink, 1966).

rem $F R \geq 1$ (Tabela 1).

As cucurbitáceas melão 'Redondo Amarelo', melão 'Gulfcoast', melão 'Chilton', melancia 'Charleston Gray', progênie da Coréia, Lagenaria siceraria, bucha Metro, B. hispida e Trichosanthes cucumerins foram considerados resistentes a $M$. javanica $\mathrm{FR}<1$ e os demais genótipos considerados suscetíveis (FR $\geq 1$ ) em 2010 (Tabela 1).

No experimento de 2011, os resultados foram semelhantes aos de 2010, com 
Tabela 2. Porcentagem de pegamento das enxertias utilizando porta-enxertos resistentes a Meloidogyne incognita e/ou Meloidogyne javanica e os melões rendilhados 'Bônus n 2' e 'Fantasy' em abril de 2011 [Percentage of grafting success using rootstocks resistant to Meloidogyne incognita and/or Meloidogyne javanica on net melons 'Bonus \# 2' and 'Fantasy' in April 2011). Jaboticabal, UNESP, 2011.

\begin{tabular}{lcc}
\hline \multirow{2}{*}{ Porta-enxertos } & \multicolumn{2}{c}{ Sucesso da enxertia (\%) } \\
\cline { 2 - 3 } & 'Bônus n' 2' & 'Fantasy' \\
\hline melão CNPH 01-962 & $99,7 \mathrm{a}$ & $98,7 \mathrm{a}$ \\
melão CNPH 01-963 & $100,0 \mathrm{a}$ & $99,7 \mathrm{a}$ \\
melão 'Gaúcho Redondo' & $100,0 \mathrm{a}$ & $100,0 \mathrm{a}$ \\
melão 'Redondo Amarelo' & $100,0 \mathrm{a}$ & $100,0 \mathrm{a}$ \\
melancia 'Charleston Gray' & $99,7 \mathrm{a}$ & $99,7 \mathrm{a}$ \\
melancia Progênie da Coreia & $100,0 \mathrm{a}$ & $98,7 \mathrm{a}$ \\
bucha Metro & $100,0 \mathrm{a}$ & $100,0 \mathrm{a}$ \\
Benincasa hispida & $100,0 \mathrm{a}$ & $100,0 \mathrm{a}$ \\
Trichosanthes cucumerins & $100,0 \mathrm{a}$ & $100,0 \mathrm{a}$ \\
\hline CV (\%) & 0,37 & 0,61 \\
\hline
\end{tabular}

Médias seguidas da mesma letra na coluna não diferem entre si pelo teste de Tukey $(p<0,05)$ [means followed by the same letter at column did not differ by Tukey test, $(\mathrm{p}<0,05)]$.

exceção do CNPH 01-930 $(\mathrm{FR}=1,0)$ para M. incognita e melão 'Gulfcoast', melão 'Chilton', Lagenaria siceraria e bucha Metro (todos com FR=1,0) para $M$. javanica, os quais no experimento do ano anterior foram considerados resistentes. Embora a temperatura seja uma característica favorável ao desenvolvimento dos nematoides, esta não pode explicar o fato desses porta-enxertos serem divergentes nos resultados nos dois anos de estudos, já que, as médias de temperatura das duas épocas foram semelhantes.

Santos et al. (1999) utilizaram uma escala de notas, conforme a presença ou não de galhas para a avaliação de resistência aos nematoides. No experimento aqui em questão, foi utilizado somente o fator de reprodução, uma vez que, as raízes dos porta-enxertos suscetíveis quando examinadas ao estereoscópio apresentaram grande massas de ovos, porém em avaliação a olho nú, as galhas eram muito pequenas, quase imperceptíveis, o que impossibilitou a contagem das mesmas.

Os porta-enxertos melão 'Gulfcoast' e 'Chilton' e a melancia 'Charleston Gray' não se diferenciaram estatisticamente pelo teste de Scott \& Knott no ano de 2011 para M. javanica, mas de acordo com a classificação de resistência proposta por Oostenbrink (1966), suscetíveis a M. incognita e resistentes a M. javanica, já as linhagens de melancia testadas por Thies et al. (2010), derivadas de Citrullus lanatus var. citroides foram resistentes a $M$. incognita e $M$. javanica.

Conforme a Tabela 1, no ano de 2010, os melões rendilhados 'Gulfcoast' e 'Chilton' foram considerados suscetíveis ao $M$. incognita, resultados semelhantes aos de Nugent \& Dukes (1997).

Não houve diferença significativa para a porcentagem de pegamento dos porta-enxertos resistentes a Meloidogyne incognita e/ou M. javanica com os melões rendilhados 'Bônus $n^{\circ}$ 2' e 'Fantasy'. Todos os porta-enxertos apresentaram taxas de enxertia acima de $98 \%$ (Tabela 2). Para o porta-enxerto bucha Metro enxertado com melão 'Bônus $\mathrm{n}^{\circ} 2^{2}$ ', o pegamento foi superior ao obtido por Rizzo et al. (2000), com $83,3 \%$ também utilizando o método de fenda-cheia.

Ito et al. (2009), avaliando porta-enxertos com resistência a Didymella bryoniae e a compatibilidade da enxertia com melão 'Bônus $n^{\circ} 2$ ', observaram que a combinação do melão rendilhado com $B$. hispida foi a mais produtiva e obteve quase $100 \%$ de pegamento, semelhante ao encontrado neste experimento. Embora não se tenha realizado os testes de produtividade, o porta-enxerto $B$. hispida foi o único utilizado resistente aos dois nematoides de galha $M$. incognita e M. javanica.

Os resultados de porcentagem de pegamento do melão 'Gaúcho Redondo', abóbora 'Goianinha' e B. hispida com melão rendilhado 'Bônus $\mathrm{n}^{\circ} 2^{\text {' }}$ foram semelhantes aos obtidos por Ito et al. (2009) com sucessos de enxertias próximos de $100 \%$.

Em uma área infestada apenas com Meloidogyne incognita, os melões rendilhados 'Bônus no 2' e 'Fantasy' serão beneficiados com as enxertias sobre os porta-enxertos CNPH 01-962, CNPH 01-963 e ‘Gaúcho redondo', já que estes porta-enxertos são resistentes ao patógeno e tiveram um bom pegamento nas enxertias. Em locais infestados apenas com Meloidogyne javanica, é indicada a utilização de melão 'Redondo Amarelo', melancia 'Charleston Gray', Progênie da Coréia e Trichosanthes cucumerins 
como porta-enxertos para melão rendilhado 'Bônus no 2' e 'Fantasy' no controle do nematoide. O porta-enxerto Benincasa hispida é indicado para enxertia com melão rendilhado 'Bônus $n^{\circ} 2$ ' ou 'Fantasy' em solos em que ocorrem as duas espécies de nematoides conjuntamente ou em separado, pois foi resistente às duas espécies. Sendo assim, pode-se recomendar o manejo do(s) nematoide(s) utilizando os porta-enxertos resistentes conforme a espécie de Meloidogyne que prevalece no local infestado.

\section{AGRADECIMENTOS}

Agradecemos à FAPESP pela concessão de bolsa de doutorado à primeira autora do trabalho (Processo $n^{\circ}$ 09/52681-6), de Auxílio à pesquisa (Processo $n^{\circ}$ 09/52682-2) e ao CNPq pela bolsa de produtividade à terceira autora.

\section{REFERÊNCIAS}

BAN SG; ZANID K; DUMICID G; RASPUDID E; BAN D. 2011. Growth and yield of grafted cucumbers in the soil infested with root-knot nematodes. In: INTERNATIONAL SYMPOSIUM ON VEGETABLE GRAFTING, 2011, Viterbo. Abstracts... Viterbo: [Universitá Degli Studi della Tuscia].

BONETTI SI; FERRAZ S.1981. Modificações do método de Hussey \& Barker para extração de ovos de Meloidogyne exigua de raízes de cafeeiro. Fitopatologia Brasileira 6, p.553 (Resumo).

CHANDRA P; SAO R; GAUTAM SK; PODDAR AN. 2010. Initial population density and its effect on the pathogenic potential and population growth of the root knot nematode Meloidogyne incognita in four species of cucurbits. Asian Journal of Plant Pathology 4: 1-15.

EISENBACK JD; HIRSCHMANN H; SASSER JN; TRIANTAPHYLLOU AC. 1981. A guide to the four most common species of rootknot nematodes (Meloidogyne spp.) with a pictorial key. Raleigh: Departments of Plant Pathology and Genetics North Carolina State University, 48p.

GOTO R; SANTOS HS; CAÑIZARES KAL. 2003. Enxertia em hortaliças. São Paulo: Editora UNESP. p.19

HARTMAN KM; SASSER JN. 1985. Identification of Meloidogyne species on the basis of differential host test and perinealpattern morphology. In: An Advanced Treatise on Meloidogyne. v2. Methodology. BARKER KR; CARTER CC; SASSER JN (eds). Raleigh, Noth Carolina. 69-77.

HUSSEY RS; BARKER KR. 1973. A comparasion of methods of collecting inocula of Meloidogyne spp., including a new technique. Plant Disease Report 57: 1025-1028.

ITO LA; CHARLO HCO; CASTOLDI R; BRAZ LT; CAMARGO M. 2009. Seleção de portaenxertos resistentes ao cancro da haste e seus efeitos na produtividade de melão 'Bônus $\mathrm{n}^{\circ}{ }^{2}$ '. Revista Brasileira de Fruticultura 31: 262-267.

LEE JM; KUBOTA C; TSAO SJ; BIE Z; ECHEVARRIA PH; MORRA L; ODA M. 2010. Current status of vegetable grafting: diffusion, grafting techniques, automation. Scientia Horticulturae 127: 93-105.

MARTIGNONI D; REDA R; ALEANDRI MP; CHILOSI G. 2011. Evaluation of response of a melon rootstock to mycorrhization with the AM Glomus intraradices in nursery. In: INTERNATIONAL SYMPOSIUM ON VEGETABLE GRAFTING, Viterbo. Abstracts... Viterbo: [Universitá Degli Studi della Tuscia]. p. 77.

NUGENT PE; DUKES PD. 1997. Root-knot nematode resistance in Cucumis species. Journal of Agricultural and Food Chemistry 45: 880-881.

OOSTENBRINK M. 1966. Major characteristics of the relation between nematodes and plants. Medelingen Van De landbouwhogeschool Te Wageningen 66: 1-46.

PINHEIRO JB; AMARO GB. 2010. Ocorrência e controle de nematoides nas principais espécies cultivadas de cucurbitáceas. Embrapa Hortaliças. Circular técnica. 7p.

RIZZO AAN; CHAVES FCM; LAURA, VA; GOTO R. 2000. Avaliação de tipos de enxertia e porta-enxertos para melão rendilhado. Horticultura Brasileira 18: 466-469.

SANTOS AA; VIDAL JC; FREIRE FCO; PAIVA WO; FREITAS ASM. 1999. Avaliação de genótipos de melão para resistência à meloidoginose e ao oídio. Embrapa Agroindústria Tropical 55: 1-3.

SILVA GS; FERRAZ S; SANTOS JM. 1989. Atração, penetração e desenvolvimento de larvas de Meloidogyne javanica em raízes de Crotalaria spp. Nematologia Brasileira 13: 151-163.

SINGUENZA C; SCHOCHOW M; TURINI T; PLOEGA. 2005. Use of Cucumis metuliferus as a rootstock for melon to manage Meloidogyne incognita. Journal of Nematology 37: 276-280.

TAYLOR AL; NETSCHER C. 1974. An improved technique for preparing perineal patterns of Meloidogyne spp. Nematologica 20: 268-269.

THIES JA; ARISS JJ; HASSELL RL; OLSEN S; KOUSIK CS; LEVI A. 2010. Grafting for management of southern root-knot nematode, Meloidogyne incognita, in watermelon. Plant Disease. 94:1195-1199. 